\title{
Parasitism of Aedes albopictus by Ascogregarina taiwanensis lowers its competitive ability against Aedes triseriatus
}

\author{
Emma Stump ${ }^{1}$, Lauren M. Childs ${ }^{2}$ and Melody Walker ${ }^{2^{*+}}$ (1)
}

\begin{abstract}
Background: Mosquitoes are vectors for diseases such as dengue, malaria and La Crosse virus that significantly impact the human population. When multiple mosquito species are present, the competition between species may alter population dynamics as well as disease spread. Two mosquito species, Aedes albopictus and Aedes triseriatus, both inhabit areas where La Crosse virus is found. Infection of Aedes albopictus by the parasite Ascogregarina taiwanensis and Aedes triseriatus by the parasite Ascogregarina barretti can decrease a mosquito's fitness, respectively. In particular, the decrease in fitness of Aedes albopictus occurs through the impact of Ascogregarina taiwanensis on female fecundity, larval development rate, and larval mortality and may impact its initial competitive advantage over Aedes triseriatus during invasion.

Methods: We examine the effects of parasitism of gregarine parasites on Aedes albopictus and triseriatus population dynamics and competition with a focus on when Aedes albopictus is new to an area. We build a compartmental model including competition between Aedes albopictus and triseriatus while under parasitism of the gregarine parasites. Using parameters based on the literature, we simulate the dynamics and analyze the equilibrium population proportion of the two species. We consider the presence of both parasites and potential dilution effects.

Results: We show that increased levels of parasitism in Aedes albopictus will decrease the initial competitive advantage of the species over Aedes triseriatus and increase the survivorship of Aedes triseriatus. We find Aedes albopictus is better able to invade when there is more extreme parasitism of Aedes triseriatus. Furthermore, although the transient dynamics differ, dilution of the parasite density through uptake by both species does not alter the equilibrium population sizes of either species.
\end{abstract}

Conclusions: Mosquito population dynamics are affected by many factors, such as abiotic factors (e.g. temperature and humidity) and competition between mosquito species. This is especially true when multiple mosquito species are vying to live in the same area. Knowledge of how population dynamics are affected by gregarine parasites among competing species can inform future mosquito control efforts and help prevent the spread of vector-borne disease.

Keywords: Mosquito population dynamics, Competition, Aedes albopictus, Aedes triseriatus, Parasitism, Ascogregarina taiwanensis, Ascogregarina barretti

\footnotetext{
*Correspondence: melody@vt.edu ${ }^{*}$

${ }^{2}$ Department of Mathematics, Virginia Tech, 460 McBryde Hall, 225

Stanger Street, 24061 Blacksburg, VA, USA

Full list of author information is available at the end of the article
}

(c) The Author(s) 2021. This article is licensed under a Creative Commons Attribution 4.0 International License, which permits use, sharing, adaptation, distribution and reproduction in any medium or format, as long as you give appropriate credit to the original author(s) and the source, provide a link to the Creative Commons licence, and indicate if changes were made. The images or other third party material in this article are included in the article's Creative Commons licence, unless indicated otherwise in a credit line to the material. If material is not included in the article's Creative Commons licence and your intended use is not permitted by statutory regulation or exceeds the permitted use, you will need to obtain permission directly from the copyright holder. To view a copy of this licence, visit http://creativeco mmons.org/licenses/by/4.0/. The Creative Commons Public Domain Dedication waiver (http://creativecommons.org/publicdomain/ zero/1.0/) applies to the data made available in this article, unless otherwise stated in a credit line to the data. 


\section{Background}

La Crosse encephalitis virus (LACV) can cause brain swelling and lead to severe neuroinvasive disease in children under 15 [1], which can cause lifelong health effects [2]. While it only leads to a few reported cases each year, it is likely under-reported. We focus on two species known to transmit LACV, Aedes albopictus and Aedes triseriatus, and the competition between them [3, 4]. Female mosquitoes of these species may become disease vectors when they feed on infected individuals and transmit the virus to other animals or humans via a second bloodmeal [5].

Aedes (Ae.) albopictus, also known as the Asian tiger mosquito, is native to subtropical regions of southeast Asia and is a common vector of arboviruses such as Dengue fever virus, West Nile virus, and La Crosse encephalitis virus [6]. The first appearance of Ae. albopictus in the USA was in Houston, Texas, in 1985 [7, 8]. Since its introduction, Ae. albopictus has spread throughout the continental US and now occupies much of the southern and eastern US, stretching from Texas to New Jersey [9]. Ae. albopictus is known for its ability to adapt to a range of climates and to be a strong competitor to other species that share larval habitats [10]. In 2001, La Crosse virus was isolated in Ae. albopictus [11]. Fourteen years later, it was estimated that 3.01 of every 1000 Ae. albopictus were infected with the La Crosse virus [12].

Ae. triseriatus, known as the eastern tree hole mosquito, is native to the eastern US. Ae triseriatus is the primary vector for the La Crosse virus [3, 4], a pathogen endemic to southwest Virginia [2,3]. Ae. albopictus will bite both small mammals and humans, making it more likely to infect human's than Ae. triseriatus, whose preference is for small mammals [4].

Several laboratory studies have shown that Ae. albopictus is the superior competitor compared to Ae. triseriatus [13-19]. In Bevins [14], they showed a 10\% drop in survival for Ae. triseriatus when a quarter of the mosquitoes present were Ae. albopictus and a $20 \%$ drop in Ae. triseriatus survival when half of the mosquitoes were Ae. albopictus. In Ho et al. [13], the authors showed that while the development time of Ae. albopictus was not significantly altered by competition, the development time of Ae. triseriatus was increased when in a shared habitat with other Aedes species. Moreover, Ae. albopictus larvae inhibit egg hatching of other species such as Ae. triseriatus, while there is no significant inhibition on their own species [15]. However, in a more recent meta-analysis, competitive equivalence of Ae. albopictus and Ae. triseriatus was suggested [20]. In a study with two different environments, they found that Ae. albopictus did much better in tires, but did worse than Ae. triseriatus in treeholes [16].
Ae. albopictus and Ae. triseriatus are parasitized by Ascogregarina (As.) taiwanensis and Ascogregarina (As.) barretti, respectively. Ascogregarina are intestinal protozoan parasites that inhabit the gut of the mosquito throughout its life cycle [7, 21]. The effects and prevalence of these parasites are heterogeneous. In the wild, typically 67 to $95 \%$ of a given population of Ae. albopictus are infected with As. taiwanensis [22]. Infection of Ae. albopictus by As. taiwanensis lengthens larval development time for both male and female mosquitoes, reduces adult female fecundity, increases larval mortality, and reduces egg laying and hatching rates $[7,23]$. However, Aliabadi and Juliano saw that mortality of Ae. albopictus was not significantly affected by As. taiwanensis [7]. For Ae. triseriatus, one study found $80 \%$ of their collected sites harbored As. barretti. Treeholes showed more infected sites than tires [24]. In contrast, another study found that only 5 and $23.6 \%$ of Ae. triseriatus are infected [25]. Development time and mortality have been seen to increase in Ae. triseriatus when infected by As. barretti [21, 25]. However, Beier and Harris [24] showed no significant effect of As. barretti on Ae. triseriatus mortality. While As. barretti impacts the fitness of Ae. triseriatus, its effects are mostly dependent on resource availability [21]. In low resource conditions they find that the Ae. triseriatus development time is increased significantly, but with sufficient resources there is not a significant difference. Another study showed a significant difference in survival of Ae. triseriatus infected with As. barretti compared to those uninfected [25].

In this work, we build a mathematical model of Aedes population dynamics accounting for parasitism of $A e$. albopictus by As. taiwanensis and Ae. triseriatus by As. barretti. Our model formulation encodes competition between the two species of Aedes using the Lokta-Volterra model, a basis for many inter-specific competition models [26-31] including mosquito populations [16, 32-34]. In Kuno [35], a two-species Lokta-Volterra competition model is introduced, which includes reproductive interference. Other competition models also show the importance of reproductive interference between $A e$. aegypti and Ae. albopictus [34, 36]. However, in contrast to the reports on reproductive interference seen with $A e$. albopictus and Ae. aegypti, there appears to be a lack of studies evaluating reproductive interference between Ae. albopictus and Ae. triseriatus. Livdahl and Willey [16] compared Ae. albopictus and Ae. triseriatus using a Lokta-Volterra model to explain how possible competition affects them in either tree holes or tires. This model was then extended to include La Crosse spread [32] and showed a lack of importance of Ae. albopictus in the recent resurgence of $\mathrm{LACV}$. 
The life cycle of gregarine parasites mimics that of their host: each stage of its development is analogous to a stage within mosquito development. See the inset in Fig. 1. Transmission of gregarine parasites is horizontal between Aedes mosquitoes individuals and does not occur from parent to offspring [22]. Initial infection occurs when the mosquito larvae ingest oocysts. After ingestion, the gregarine parasite travels through the midgut, epithelial tissues, and excretory system of the mosquito, transitioning into different life stages as the mosquito matures to adulthood. From there, the parasite reproduces and offspring are excreted by the adult mosquitoes into breeding containers [37, 38].

Since new Ae. albopictus habitats are unlikely to be contaminated with As. taiwanensis, new populations of Ae. albopictus can initially escape parasitism. This lack of parasite infection in newly established populations gives the mosquito a potential competitive advantage over Ae. triseriatus [7, 22]. Knowledge of how parasite infection affects the survivorship of Ae. albopictus will provide insight into the competition between the two mosquito species and subsequently help to deepen understanding of the spread of mosquito-borne infectious disease. In this study, we examine whether the parasitism of $A e$. albopictus by As. taiwanensis decreases the mosquito's competitive ability enough to alter the dynamics between the two Aedes mosquitoes. We build a compartmental model of the interactions of the two mosquito species and the effect of the parasites As. taiwanensis and As. barretti. We parameterize the model from published literature, simulate dynamics, investigate a wide parameter space, and examine the effects of parasitism.

\section{Methods}

We constructed a four-compartment model of mosquito population dynamics including larval and adult stages of both Ae. albopictus and Ae. triseriatus (Eq. 1). We simplify the life cycle of the Aedes mosquito to just two stages because most, if not all, of the effects of competition and parasitism are felt at the larval and adult stages. Furthermore, including all mosquito life stages would unnecessarily complicate the model. We based our model of competition on the Lotka-Volterra model of species interaction [39-42]. We use the Lokta-Volterra model to incorporate inter-specific competition between the two species and include effects of parasitism. We assume a fixed level of parasitism in Ae. triseriatus, impacting development time and mortality. We consider two conditions: either a high or low effect of As. barretti. To model the effect of different As. taiwanensis parasite levels as Ae. triseriatus becomes established, we vary the level parasitism. We incorporate the effect of parasitism on Ae. albopictus in three ways: (i) decreased fecundity, (ii) increased

\section{Aedes mosquito Lifecycle with Ascogregarina infection}

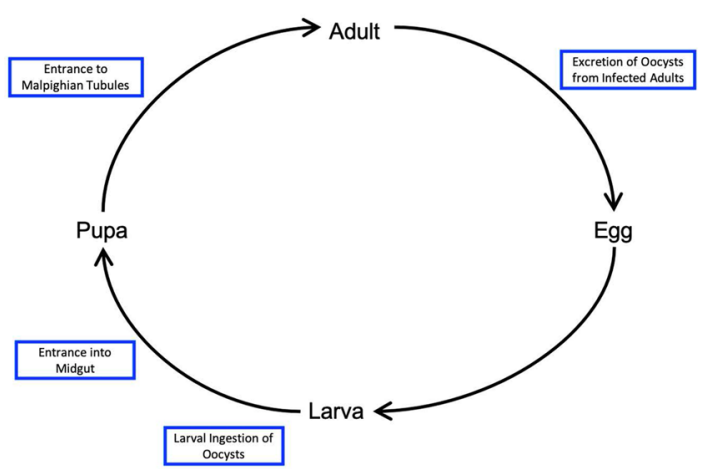

Fig. 1 Life cycle of the mosquito and parasite. Interaction between life cycles of Aedes mosquito, shown as the black lines, and Ascogregarina parasite, shown as the blue boxes. As the mosquito progresses from stage to stage in its life cycle, its gregarine parasite progresses through in a mirrored fashion. For details on the parasite life cycle, see Chen [37]

development time, and (iii) increased larval mortality. This provides a potential decrease in the competitive advantage of Ae. albopictus over the population of Ae. triseriatus as Ae. albopictus becomes established in a new area.

The model includes four compartments: larval Ae. albopictus $\left(L_{a}\right)$, adult Ae. albopictus $\left(A_{a}\right)$, larval Ae. triseriatus $\left(L_{t}\right)$, and adult $A e$. triseriatus $\left(A_{t}\right)$. Larval mosquitoes $L_{i}$ are born at rate $\beta_{i}$, die at rate $\mu_{L_{i}}$, and develop at rate $\delta_{i}$ where $i \in\{a, t\}$. Adults emerge from larva at rate $\delta_{i}$ and die at rate $\mu_{A_{i}}$ where $i \in\{a, t\}$ for Ae. albopictus and Ae. triseriatus, respectively. Similar formulations for Ae. albopictus population dynamics are found in [43]. The parameters $\rho_{a}$ and $\rho_{t}$ represent the proportion of adults that are female. $K$ is the carrying capacity of the larval population for the two species. The Lotka-Volterra competition coefficients, $\alpha_{a}$ and $\alpha_{t}$, quantify the intrinsic effect of one species on the other. Parasitism for Ae. albopictus is included through the parameters representing effects on female fecundity $\left(\gamma_{b_{a}}\right)$, larval development rate $\left(\gamma_{d_{a}}\right)$, and larval mortality $\left(\gamma_{m_{a}}\right)$. The parasite parameters for Ae. triseriatus are larval development rate $\left(\gamma_{d_{t}}\right)$ and larval mortality $\left(\gamma_{m_{t}}\right)$. This forms our system of ordinary differential equations:

$$
\begin{aligned}
\frac{d L_{a}}{d t} & =\frac{\beta_{a}}{\gamma_{b_{a}}} \rho_{a} A_{a}\left(1-\frac{L_{a}+\alpha_{t} L_{t}}{K}\right)-\frac{1}{\gamma_{d_{a}} \delta_{a}} L_{a}-\gamma_{m_{a}} \mu_{L_{a}} L_{a}, \\
\frac{d A_{a}}{d t} & =\frac{1}{\gamma_{d_{a}} \delta_{a}} L_{a}-\mu_{A_{a}} A_{a} \\
\frac{d L_{t}}{d t} & =\rho_{t} \beta_{t} A_{t}\left(1-\frac{\alpha_{a} L_{a}+L_{t}}{K}\right)-\frac{1}{\gamma_{d_{t}} \delta_{t}} L_{t}-\gamma_{m_{t}} \mu_{L_{t}} L_{t}, \\
\frac{d A_{t}}{d t} & =\frac{1}{\gamma_{d_{t}} \delta_{t}} L_{t}-\mu_{A_{t}} A_{t} .
\end{aligned}
$$


From a literature search, we determined biologically relevant ranges for parameter values. A description of the parameters is provided in Table 1. As it was often difficult to directly find relevant values in the literature, we transformed values found to meet our parameter descriptions. The birthrate was calculated from data for the gross reproductive rate (GRR) and length of gonotrophic cycle (GC) by taking the minimum GRR and dividing by the maximum length of the GC. The maximum value was found by dividing the maximum GRR by the minimum GC length [44]. With this, we calculated a range of 2.5-56 eggs laid per adult female per day. A similar calculation was performed to find a birthrate for Ae. triseriatus, where we calculated a range of 3-26 eggs laid per adult female per day $[45,46]$. Development time can vary based on a variety of factors and has been found to be as short as 9 days and as long as 40 days for Ae. albopictus [13, 47, 48]. Ae. triseriatus has been shown to develop more slowly than Ae. albopictus [13, 18]. In the model, these values are incorporated directly as development time of larvae, $\left(\delta_{a}\right)$ and $\left(\delta_{t}\right)$. Larval $\left(\mu_{L_{a}}\right)$ and adult $\left(\mu_{A_{a}}\right)$ mortality for Ae. albopictus were calculated to be 0.067 and 0.05 , respectively, from survival rates and development rates [13, 44, 47,48], such that $\mu=1-$ ( survival rate $)^{(1 / \delta)}$. For Ae. triseriatus, larval $\left(\mu_{L_{t}}\right)$ and adult $\left(\mu_{A_{t}}\right)$ mortalities were found to be 0.009 and 0.1 , respectively $[49,50]$. The carrying capacity for the larval population of both species $(K)$ was set at 60 as was used for a study performed in $200 \mathrm{ml}$ of water [7]. It is important to note that this number is relative and could be scaled to fit different size larval containers. In this study we focus on the proportion of each species such that raw population sizes are not influential. There is evidence of potential sex imbalance among mosquitoes especially under low food resources. Thus, for Ae. triseriatus, we set the proportion of females, $\rho_{t}$, to be between 0.2 and 0.6 [17]. In the same study, Ae. albopictus showed less variation, $0.4-0.55$, in the proportion females, so we set $\rho_{a}$ accordingly.

In Livdahl and Willey [16], the competition parameters from the Lokta-Volterra model were fit using nutrient fluids. The competition parameter of Ae. albopictus on Ae. triseriatus $\left(\alpha_{a}\right)$ and the competition parameter of Ae. triseriatus on Ae. albopictus $\left(\alpha_{t}\right)$ were found to be 0.42 and 0.73 in treehole fluid and 0.83 and 0.25 in tire fluid, respectively. Many factors dictate which species will be the better competitor; however, as we considered that Ae. albopictus is generally the greater competitor, we chose a range of $[0,0.75]$ for the effect on Ae. albopictus from Ae. triseriatus and a range of $[0.4,1]$ for the effect on Ae. triseriatus from Ae. albopictus.

The effects of parasitism for Ae. albopictus on female fecundity $\left(\gamma_{b_{a}}\right)$, larval development rate $\left(\gamma_{d_{a}}\right)$, and larval mortality $\left(\gamma_{m_{a}}\right)$ were shown to vary with resource availability and environmental context [7, 23, 25, 51]. In Comiskey et al. [23], they found that in low resource conditions the mortality of infected Ae. albopictus larvae was seven times greater than in uninfected larvae. They also found that fecundity and fertility were reduced by $>20 \%$ and development time was increased by $44 \%$. In Aliabadi and Juliano [7], they showed that the development time of infected Ae. albopictus increases with greater interspecific competition with Ae. triseriatus, whereas without Ae. triseriatus, the median development time of infected Ae. albopictus was not significantly different. They also found that survival rates were significantly different for Ae. triseriatus at lower densities with Ae. albopictus. They did not observe a significant change in survival for Ae. albopictus from parasitism, but a greater effect from intra- and inter-specific competition. Ae. triseriatus was found in one study to have as much as a $2.8-3.5$ greater death rate when infected [25]. In a relatively recent study by Soghigian and Livdahl [51], they showed that in the absence of parasite infection, survival of Ae. albopictus is about $98 \%$, but with the greatest amount of infection observed, the survival decreases to approximately $71 \%$. This constitutes a 15 times greater mortality rate. In another study by Walker et al. [21], they found an increase in development time for Ae. triseriatus in the laboratory with low resources, but not in the field experiment.

We initially set all Ae. albopictus parasite parameter values to 1 , which represents the case with no parasitism. We then consider ranges from 1 to 2 for a parasite's effect on fecundity and development time and 1-16 for mortality. These values were chosen to cover the ranges of changes found in $[23,51]$. We will extend the ranges of the parasite parameters for development time and fecundity in further analyses. We are assuming that $A e$. triseriatus is already infected, so we initially fix these parameter values as $\gamma_{d_{t}}=1.2$ and $\gamma_{m_{t}}=1.5$ to be a relatively small effect. We also consider a higher effect to parasitism with $\gamma_{d_{t}}=2$ and $\gamma_{m_{t}}=4$ as a comparison. If $\gamma_{d_{a}}=5$, this would mean that the development time was five times greater when Ae. albopictus is infected with its parasite. If any of the parasite parameters are set to 1 this indicates no effect of parasitism.

We simulated the mosquito population dynamics from our ODE model in Matlab. Fixed parameter values, shown in Table 1, were used in these simulations. The initial conditions used for all simulations were $L_{a}=30, A_{a}=0, L_{t}=30$, and $A_{t}=0$. We use these initial conditions for consistency with Aliabadi and Juliano [7], but as we run the simulation until equilibrium other initial values will give the same results. Simulations were 
Table 1 Parameter values and Latin hypercube sampling ranges

\begin{tabular}{|c|c|c|c|c|c|}
\hline \multicolumn{6}{|c|}{ Aedes albopictus } \\
\hline Symbol & Description & Value & LHS range & Units & Reference \\
\hline$\beta_{a}$ & Birth Rate & 32.6 & $2.5-56$ & Eggs/day & {$[44]$} \\
\hline$\delta_{a}$ & Development Time & 10 & $9-45$ & $1 /$ days & {$[13,47,48]$} \\
\hline$\mu_{L_{a}}$ & Larval mortality & 0.067 & $0.005-0.4$ & 1/day & {$[13,44]$} \\
\hline$\mu_{A_{a}}$ & Adult mortality & 0.045 & $0.01-0.065$ & 1/day & {$[44,47,48]$} \\
\hline$\alpha_{a}$ & Competition parameter & $0.83,0.42$ & $0.4-1$ & Unitless & {$[16]$} \\
\hline$\rho_{a}$ & Adult female proportion & 0.5 & $0.2-0.6$ & Unitless & {$[17]$} \\
\hline \multicolumn{6}{|c|}{ Aedes triseriatus } \\
\hline$\beta_{t}$ & Birth rate & 11 & $3-26$ & Eggs/day & {$[45,46,50]$} \\
\hline$\delta_{t}$ & Development time & 22 & $13-55$ & $1 /$ days & {$[12,13,18]$} \\
\hline$\mu_{L_{t}}$ & Larval mortality & 0.009 & $0.002-0.011$ & 1/day & {$[13,17,48]$} \\
\hline$\mu_{A_{t}}$ & Adult mortality & 0.1 & $0.03-0.1$ & 1/day & [49] \\
\hline$\alpha_{t}$ & Competition parameter & $0.25, .73$ & $0-0.75$ & Unitless & {$[16]$} \\
\hline$\rho_{a}$ & Adult female proportion & 0.5 & $0.4-0.55$ & Unitless & {$[17]$} \\
\hline \multicolumn{6}{|c|}{ Other parameters } \\
\hline$\gamma_{b_{a}}$ & Effect on fecundity & 1.0 & $1-2$ & Unitless & Varied \\
\hline$\gamma_{d_{a}}$ & Effect on development rate & 1.0 & $1-2$ & Unitless & Varied \\
\hline$\gamma_{m_{a}}$ & Effect on larval mortality & 1.0 & $1-16$ & Unitless & Varied \\
\hline$\gamma_{d_{t}}$ & Effect on development rate (Ae. Triseriatus) & $1.2,2$ & Fixed & Unitless & Varied \\
\hline$\gamma_{m_{t}}$ & Effect on larval mortality (Ae. Triseriatus) & $1.5,4$ & Fixed & Unitless & Varied \\
\hline K & Carrying capacity & 60 & Fixed & Number of larvae & {$[16]$} \\
\hline
\end{tabular}

run for 2000 days, long enough for the population of $A e$. albopictus and Ae. triseriatus larvae and adults to reach equilibrium. To begin, we considered two competition scenarios. The first was the environment within a tire $\left(\alpha_{a}=0.83, \alpha_{t}=0.25\right)$, which indicates that Ae. albopictus has a greater effect on Ae. triseriatus. In the second scenario, the environment in a treehole $\left(\alpha_{a}=0.42\right.$, $\left.\alpha_{t}=0.73\right)$, such that Ae. triseriatus, has a greater effect on Ae. albopictus. These parameters were from fitted data in Livdahl and Willey [16].

We performed a parameter sweep by sampling ranges of the parameters using Latin Hypercube Sampling (LHS) in Matlab [52]. We conducted this with a sample size of 100,000, and sampled 12 of the parameters: $\beta_{a}$, $\beta_{t}, \delta_{a}, \delta_{t}, \mu_{L_{t}}, \mu_{A_{t}}, \mu_{L_{a}}, \mu_{A_{a}}, \alpha_{t}, \alpha_{a}, \rho_{t}$, and $\rho_{a}$. Values for parasitism on Ae. albopictus $\left(\gamma_{b_{a}}, \gamma_{d_{a}}, \gamma_{m_{a}}\right)$ were fixed at 1 , and $K$ was fixed at 60 . The LHS was completed twice, first with the parasite parameters for Ae. triseriatus set at $\gamma_{d_{t}}=1.2$ and $\gamma_{m_{t}}=1.5$ and then again with them set at $\gamma_{d_{t}}=2$ and $\gamma_{m_{t}}=4$. With the results generated by the LHS, we generated a histogram reflecting the type of outcomes for each of the samples. We categorized the results into seven categories, described in Table 2. These categories were based on the proportion of the population occupied by Ae. albopictus as follows: in category (1), a proportion of $<0.01$; in category (2), a proportion of 0.01 to 0.2 ; in category (3), a proportion between 0.2 and 0.4 ; in category (4), a proportion between 0.4 and 0.6 ; in category (5), a proportion between 0.6 and 0.8 ; in category (6), a proportion between 0.8 and 0.99 ; in category (7) a proportion $>0.99$. We than did a sensitivity analysis on the parameters to account for uncertainty and variation in parameters by the partial rank correlation coefficient [53].

After sampling the space of the 12 parameters in the model in the absence of parasitism in Ae. albopictus, we focused on investigating the effects of the parasite. We repeated the LHS including the parasite parameters for Ae. albopictus. We also performed sensitivity analysis on the 100,000 samples, which included varied parasite effects. The mortality and fecundity parasite parameters were varied from 1 to 4 , and the development time parasite parameter varied from 1 to 16 , where a value of 1 meant the parasite has no effect and a value of 3 meant a three-fold increase of the associated parameter value. This means that mortality or development time is increased or fecundity is decreased compared to the value without parasitism. 
To examine the varying effects of parasitism, all parameters were fixed as listed in Table 1 except we varied the three Ae. albopictus parasite parameters with Ae. triseriatus fixed at either a high or low parasite effect. We compared effects on female fecundity, larval development rate, and larval mortality to see how these different combinations led to different outcomes in terms of the proportion Ae. albopictus in the final population. We extended the range of the parasite parameters so that all were varied from 1 to 10 .

\section{Dilution effects}

A recent study by Westby et al. [54] showed that with $A e$. japonicus and Ae. triseriatus there was a dilution effect on the amount of parasitism. Ae. japonicus decreased the number of As. barretti by consuming the parasite and not propagating it [54]. We consider this idea with the twospecies model, by allowing the parasitism parameters to have decreased effect in the presence of greater proportions of the competing species, a so-called 'dilution' effect. We modify the model by making all parasite parameters a function of the proportion of the total population. Specifically, each parasite parameter will linearly decrease from the maximum value $\gamma_{i_{\max }}$ to 1 . This is captured by:

$$
\gamma_{i}(p)=\left(\gamma_{i_{\max }}-1\right) p+1
$$

where $p$ is the proportion of a species. We choose a linear function for simplicity. Notice that if $p=1, \gamma_{i}=\gamma_{i_{\max }}$, which indicates that if a particular species is $100 \%$ of the population, then their parasite parameters will be at its maximum. If $p=0, \gamma_{i}=1$, this means that as the species goes to $0 \%$ of the population, the parasite effect of the parameter will linearly decrease to no parasite effect. In Westby et al. [54], they see a large decrease in the amount of parasitism $(\approx 82 \%$ reduction), so we allow parasitism parameters to approach 1 as the proportion of larvae goes to zero. Recall that, when the parasite parameters equal 1 , this represents no effect of parasitism. Thus, our revised system of equations becomes:

$$
\begin{aligned}
\frac{d L_{a}}{d t}= & \frac{\beta_{a} \rho_{a} A_{a}}{\gamma_{b_{a}}\left(P_{a}\right)}\left(1-\frac{L_{a}+\alpha_{t} L_{t}}{K}\right) \\
& -\frac{1}{\gamma_{d_{a}}\left(P_{a}\right) \delta_{a}} L_{a}-\gamma_{m_{a}}\left(P_{a}\right) \mu_{L_{a}} L_{a} \\
\frac{d A_{a}}{d t}= & \frac{1}{\gamma_{d_{a}}\left(P_{a}\right) \delta_{a}} L_{a}-\mu_{A_{a}} A_{a}, \\
\frac{d L_{t}}{d t}= & \rho_{t} \beta_{t} A_{t}\left(1-\frac{\alpha_{a} L_{a}+L_{t}}{K}\right) \\
& -\frac{1}{\gamma_{d_{t}}\left(P_{t}\right) \delta_{t}} L_{t}-\gamma_{m_{t}}\left(P_{t}\right) \mu_{L_{t}} L_{t}, \\
\frac{d A_{t}}{d t}= & \frac{1}{\gamma_{d_{t}}\left(P_{t}\right) \delta_{t}} L_{t}-\mu_{A_{t}} A_{t} .
\end{aligned}
$$

where $P_{a}=\frac{L_{a}}{L_{a}+L_{t}}$ and $P_{t}=\frac{L_{t}}{L_{a}+L_{t}}$.

We then consider four cases by choosing different maximum parameters for parasitism, $\gamma_{i_{\max }}$. These combination are: Ae. albopictus and Ae. triseriatus both have minor effects of parasitism, both have more severe effects, and only one has a severe effect and the other minor effect. See Table 3 for specific choices for maximum parasite parameters.

\section{Results}

To begin, we model the population dynamics of $A e$. albopictus and Ae. triseriatus without parasitism. We consider two separate environments: tire and treehole. The difference between the two scenarios is the LoktaVolterra competition parameters $\left(\alpha_{t}\right.$ and $\left.\alpha_{a}\right)$ that were fit for each environment from Livdahl and Willey [16]. In both cases, the populations settle to an equilibrium. In the tire environment, the population ends in category 6, in which Ae. albopictus dominates, but Ae. triseriatus remains at low levels (Fig. 2, right). After 150 days, we find 126 adult Ae. albopictus compared to about 5 adult Ae. triseriatus. For the treehole environment, the population ends in category 5 (Fig. 2, left), where Ae. albopictus is still the dominant species, but Ae. triseriatus has a sizeable population. After 150 days, there are approximately 54 Ae. albopictus and 19 Ae. triseriatus. Parameter values

Table 2 Categorized outcomes ${ }^{\sharp}$ Criteria is proportion of Ae. albopictus adults

\begin{tabular}{llr}
\hline Category & Description & Criteriä $\left(\boldsymbol{A}_{a}\right)$ \\
\hline 1 & Ae. triseriatus completely dominates, Ae. albopictus wiped out & $A_{a}<0.01$ \\
2 & Ae. triseriatus dominates, few Ae. albopictus remain & $0.01<A_{a}<0.15$ \\
3 & Ae. triseriatus more present, Ae. albopictus still persists & $0.20<A_{a}<0.40$ \\
4 & Both species coexist in even proportions & $0.40<A_{a}<0.60$ \\
5 & Ae. albopictus more present, Ae. triseriatus persists & $0.60<A_{a}<0.80$ \\
6 & Ae. albopictus dominates, few Ae. triseriatus remain & $0.80<A_{a}<0.99$ \\
7 & Ae. albopictus completely dominates, Ae. triseriatus wiped out & $A_{a}>0.99$ \\
\hline
\end{tabular}


are the estimated averages from the literature and are found in Table 1.

To account for known variability in parameters, we examine dynamics across a broad parameter space using a LHS with the ranges indicated in Table 1. Assuming no effects of parasitism in Ae. albopictus and a low effect in Ae. triseriatus, we find that the majority, 56\%, of simulations fall into category 6 (Fig. 2a), where Ae. albopictus strongly dominates. This was the same result as in the tire scenario. In addition, approximately $25 \%$ of the simulations fall into the same category as the treehole scenario (category 5). Only $3.5 \%$ of the total 100,000 samples lead to categories in which Ae. triseriatus has a larger population than Ae. albopictus (categories 1, 2, and 3). A further $10.5 \%$ of the simulations fall into coexistence with approximately equal amounts of both species (category 4). For a small number of simulations (5\%), Ae. albopictus completely eliminated Ae. triseriatus without parasitism (category 7), but the most common scenario was for $A e$. albopictus to be the dominant species and strongly limit the population of Ae. triseriatus.

If we increase the effect of parasitism in Ae. triseriatus, we find that there is a strong shift in scenarios to where Ae. albopictus is the dominant species (Fig. 2c). In this case, approximately $66 \%$ of parameters selected fall into category 6 (few Ae. triseriatus) and 9.9\% of situations lead to elimination of Ae. triseriatus (category 7). Ae. triseriatus only has a greater proportion than Ae. albopictus in approximately $1 \%$ of the parameters sampled.

\section{Including parasitism in Ae. albopictus}

When we include the effects of parasitism for Ae. albopictus in the dynamics, we greatly increase the parameter space where Ae. triseriatus is the dominant species. We use LHS but include the parameters for parasitism: $\gamma_{d_{a}}$, $\gamma_{m_{a}}$, and $\gamma_{b_{a}}$. We find that results falling in categories 1-4 have greatly increased while those in categories 6 and 7 have significantly decreased (Fig. 2 b, d). In fact, Ae. triseriatus completely eliminates (category 1) Ae. albopictus in approximately $15.0 \%$ and $15.3 \%$ of the simulations for low and high Ae. triseriatus parasitism, respectively. However, in $30.7 \%$ and $24.4 \%$ of the simulations Ae. triseriatus is the dominant species (categories 1,2,3), and the sum of all categories where Ae. albopictus will be the dominant species is reduced to $54.7 \%$ and $64.8 \%$ (sum of categories 5, 6, 7) with low and high parasitism for $A e$. triseriatus, respectively. The total simulations with outcomes in categories 5, 6, and 7 are similar to the amount in only category 6 without Ae. albopictus parasitism. With parasitism of Ae. albopictus, category 6 still has the most outcomes, but the outcomes spread out. Overall, we see that without parasitism Ae. albopictus will be the dominant vector; however, once parasitism takes place Ae. triseriatus can become dominant.

To further examine the effects of parasitism, we vary the level of each of the three parasitism parameters. We perform these variations pairwise under four environmental scenarios: tire (Fig. 3, quadrants I and IV) and tree hole (Fig. 3, quadrants II and III) with both a low and high parasite effect on Ae. triseriatus. Recall the difference between the two scenarios is the competition parameters $\left(\alpha_{t}\right.$ and $\left.\alpha_{a}\right)$. In the tire scenario, the competition effect of Ae. albopictus on Ae. triseriatus is greater $\left(\alpha_{a}=0.83>0.25=\alpha_{t}\right)$, and for the treehole scenario, the competition effect of Ae. triseriatus on Ae. albopictus is greater $\left(\alpha_{a}=0.42<0.73=\alpha_{t}\right)$. All other parameters are fixed (Table 1). The colors in these images correspond to the colors of the seven categories from Table 2, although only six colors appear as category 7 never occurs in these simulations. Parasitism effects were varied pairwise. First, parasite effects on larval mortality $\left(\gamma_{m_{a}}\right)$ and larval development time $\left(\gamma_{d_{a}}\right)$ were varied with three constant levels of the effect on female fecundity $\left(\gamma_{b_{a}}=1,3,5\right)$ (Fig. 3). Then, parasite effects on female fecundity $\left(\gamma_{b_{a}}\right)$ and larval development time $\left(\gamma_{d_{a}}\right)$ were varied with three constant levels of the effect on mortality $\left(\gamma_{m_{a}}=1,3,5\right)$. Finally, parasite effects on larval mortality $\left(\gamma_{m_{a}}\right)$ and female fecundity $\left(\gamma_{b_{a}}\right)$ were varied with three constant levels of the effect on larval development time $\left(\gamma_{m_{a}}=1,3,5\right)$. We present these results in Additional file 2 as they are very similar to those found from fecundity versus development time.

In the tire scenario $\left(\alpha_{a}=0.83>0.25=\alpha_{t}\right)$, we find that in large portions of the parameter space Ae. albopictus strongly dominates, i.e. category 6 (Fig. 3, quadrants I and IV, dark yellow). When the effect on female fecundity $\left(\gamma_{b_{a}}\right)$ is fixed at 1 , the transition to a greater level of coexistence between the mosquito species is only seen at higher levels of effects on the larval development rate $\left(\gamma_{d_{a}}\right)$, with values around $\gamma_{d_{a}}>4$ (Fig. 3b, quadrant I and IV). For female fecundity $\left(\gamma_{b_{a}}\right)$ fixed at three and five (Fig. 3d, f, quadrant I and IV), development time effects as low as $\gamma_{d_{a}} \approx 2$ result in a shift to a low proportion of Ae. albopictus (category 5 green). For Ae. triseriatus to be the dominant species, there must be very high effects of all three parasitism parameters affecting Ae. albopictus, regardless of Ae. triseriatus' level of parasitism.

Parasite effects on the development time have more significant effects than on mortality or fecundity. In the tire scenario, this is most apparent when there is no change in the development time (Fig. 3a); then, Ae. albopictus always strongly dominates. Additionally, there is no change in category, regardless of the strength of the effect on mortality and fecundity or parasitism on Ae. triseriatus. In the tire scenario, if the effect on development 


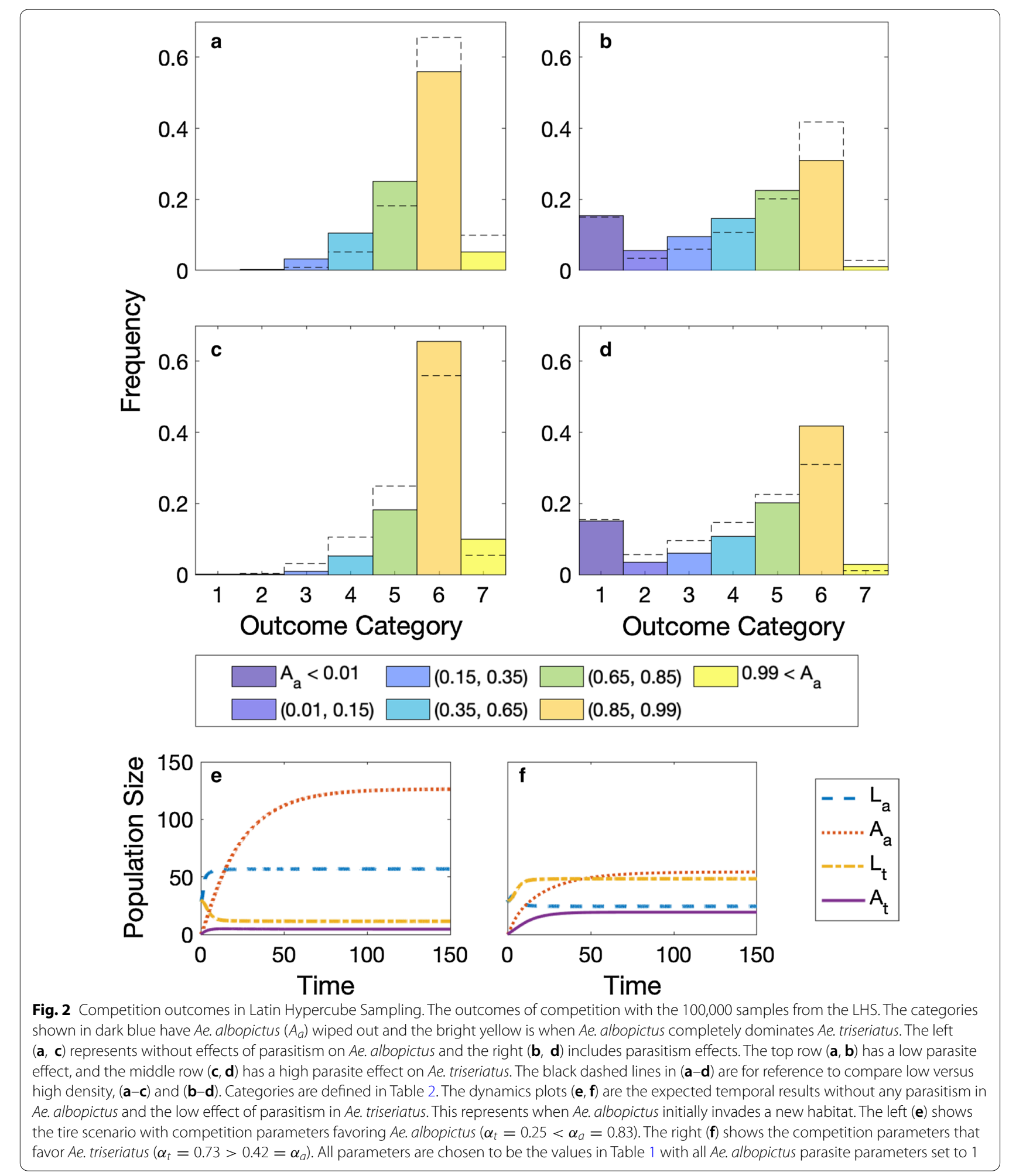


Table 3 Dilution parasitism parameters. Each level variation for parasitism (high or low) for each species

\begin{tabular}{|c|c|c|c|c|c|c|}
\hline Ae. albopictus & $\max \boldsymbol{\gamma}_{\boldsymbol{m}_{a}}$ & $\max \gamma_{d_{a}}$ & $\max \boldsymbol{\gamma}_{\boldsymbol{b}_{\boldsymbol{a}}}$ & Ae. triseriatus & $\max \boldsymbol{\gamma}_{\boldsymbol{m}_{t}}$ & $\max \gamma_{d_{t}}$ \\
\hline Low & 1.5 & 1.2 & 1.2 & Low & 1.5 & 1.2 \\
\hline High & 16 & 4 & 4 & Low & 1.5 & 1.2 \\
\hline Low & 1.5 & 1.2 & 1.2 & High & 8 & 4 \\
\hline High & 16 & 4 & 4 & High & 8 & 4 \\
\hline
\end{tabular}

time is increased by three times (Fig. 3c), effects on both fecundity and mortality must be relatively high to obtain higher levels of Ae. triseriatus. Furthermore, Ae. triseriatus will only dominate if parasite effects on both fecundity and mortality are $>10$. When the development time in tires is five times greater, only a small effect by the other two parameters is needed to decrease the proportion of Ae. albopictus to category 5. Similarly, with fixed fecundity, when the parasite increases the development time seven fold, we see that regardless of the other two parameters, the proportion of Ae. albopictus decreases to a minimum of category 5 (Fig. 3f, green and blue).

In the treehole scenario, Ae. triseriatus has a more competitive effect on Ae. albopictus. As seen with the absence of parasitism for Ae. albopictus with a low effect of parasitism on Ae. triseriatus, the default is category 5 (Fig. 3a, b, quadrant III, green in bottom left corner), in which Ae. albopictus is still the dominant species, but there are more Ae. triseriatus. In this situation, the effect on development time must only increase two-fold for the proportion of Ae. albopictus to decrease to category 4 (Fig. 3b, quadrant III, teal), in which there are relatively similar amounts of both species. When the effect on fecundity is set to 3 (Fig. 3d, quadrants II, III) and the effect of development time is $>4$, Ae. triseriatus will be the dominant species (all three shades of blue). When the effect of fecundity is set to 5 (Fig. 3f, quadrants II, III) and the development time is $>3$, Ae. triseriatus will dominate. A similar result is seen when the development time is five times greater (Fig. 3e, quadrants II, III). For all values of the other two parameters, Ae. triseriatus is the dominant species. In fact, almost half of the parameter space considered falls into category 1 where Ae. albopictus is eliminated (darkest blue). Without any parasite effects on development time (Fig. 3a, quadrants II, III), there is only a chance for the two species to be relatively equal if both other parameters are $>7$ and there is a low effect of parasitism on Ae. triseriatus.

Overall, in tires, only with very high effects on all three parameters do we find that Ae. albopictus is dominated by Ae. triseriatus, while in treeholes, we find that less parasitism is needed to eliminate Ae. albopictus, i.e. only high effects on two of the Ae. albopictus parasite parameters. In both scenarios, as we increase the effects of the parasite parameters we see that the proportion of Ae. albopictus decreases. The change is most notable as the parasite effect on Ae. albopictus development time is increased, regardless of the scenario.

\section{Importance of parameters}

From the parameters selected using LHS with a low effect of parasitism on Ae. triseriatus, we looked at the partial rank correlation coefficient (PRCC) for each parameter with respect to the final proportion of Ae. albopictus at 2000 days. In the case without parasitism, we find that all parameters have a significant impact on the model (Fig. 4, top row). When parasitism is included (Fig. 4, bottom row), the parameters which are not significant are all related to Ae. triseriatus: death of larvae, $\mu_{L_{t}}$, and proportion of females, $\rho_{t}$.

In the case without parasitism, the parameter with the most influence (largest PRCC value in magnitude) is the competition parameter $\alpha_{a}$, which is the inter-specific competition effect on Ae. triseriatus from Ae. albopictus. With a PRCC value of 0.8469 , the more $\alpha_{a}$ increases, the greater the proportion of Ae. albopictus is. Several parameters have a fairly large correlation. The following parameters all have a PRCC value $>0.5$ in magnitude (in the order of the greatest magnitude to lowest): death rate of adult Ae. albopictus, $\mu_{A_{a}}$; Ae. albopictus larval development time, $\delta_{a} ;$ Ae. triseriatus larval development time, $\delta_{t}$, the competition parameter $\alpha_{t}$ on Ae. albopictus; and death rate of adult Ae. triseriatus, $\mu_{A_{t}}$. We see that the most important parameters are the death rates of adults, transition rate to adults, and Lokta-Volterra competition parameters.

When we introduce the parasitism parameters into the LHS, we see that the parasite's effect on development time $\gamma_{m_{a}}$ has the sixth greatest impact, but the largest of all the parasitism parameters. With a PRCC of -0.1359 , we expect the increase of $\gamma_{m_{a}}$ to decrease the proportion of Ae. albopictus. The death of adult Ae. albopictus, Ae. albopictus larval development time $\delta_{a}$, Ae. albopictus birth rate $\beta_{a}$, Lokta-Volterra competition parameter $\alpha_{t}$, and death of Ae. albopictus larvae all have a magnitude of PRCC values greater than $\gamma_{d_{a}}(-0.2525,-0.2081$, $0.1754,-0.1517$, and -0.1581 , respectively). While less important than $\gamma_{m_{a}}$, the other two parasite parameters 

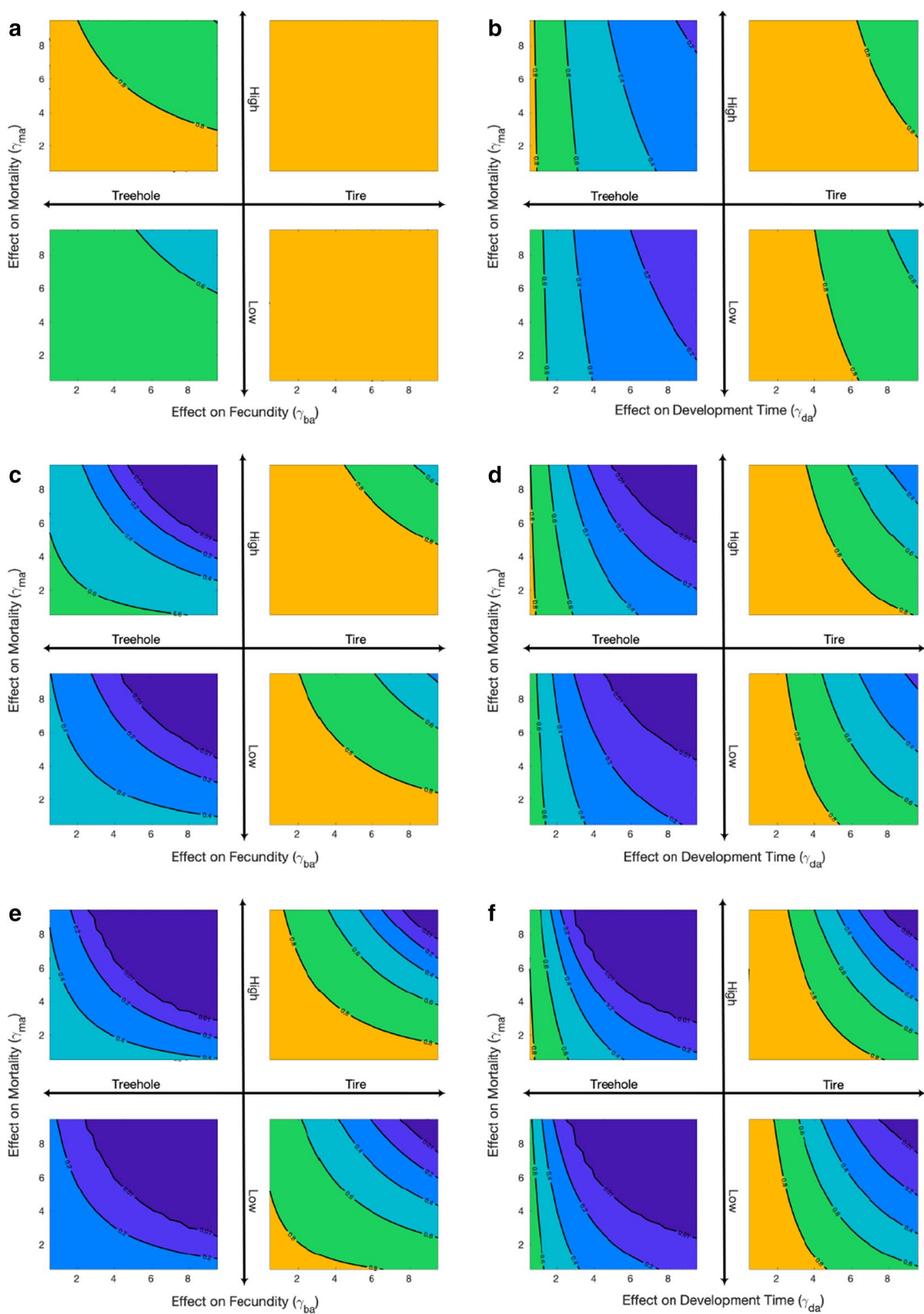

Fig. 3 Effects of parasitism on Ae. albopictus proportions. The proportion of Ae. albopictus when varying parasitism parameters. For all subplots, the right quadrants (I and IV) are the tire environment where there is a greater competitive effect on Ae. triseriatus than on Ae. albopictus; the left quadrants (II and III) are the treehole environment where the reverse is true; the upper quadrants (I and II) have high parasite effects on Ae. triseriatus; the lower quadrants (III and IV) have a low parasite effect on Ae. triseriatus. All three Ae. albopictus parasitism parameters $-\gamma_{b_{a^{\prime}}} \gamma_{m_{a^{\prime}}}$ and $\gamma_{d_{a}}$-are varied. All subplots vary mortality on the $y$-axis while the left subplots $(\mathbf{a}, \mathbf{c}, \mathbf{e})$ have fecundity on the $\mathbf{x}$-axis, and the right subplots $(\mathbf{b}, \mathbf{d}, \mathbf{f})$ have development time on the $x$-axis. Each row fixes the third parasite parameter as not varied: $1(\mathbf{a}, \mathbf{b}), 3(\mathbf{c}, \mathbf{d}), 5(\mathbf{e}$, $\mathbf{f})$. Lines distinguish between different outcome categories. For example, yellow is when the proportion of Ae. albopictus exceeds 0.8 
are statistically significant $(p<0.00001)$ as well. The PRCC value for the parasite's effect on development time is -0.1039 , and for the parasite's effect on fecundity is -0.05 .

When we consider the LHS with a high effect of parasitism on Ae. triseriatus, all parameters have a much lower effect, as indicated by the lower magnitude of the PRCC value. The parameter with the greatest magnitude PRCC (0.1666) is the Lokta-Volterra competition parameter $\alpha_{a}$; this is the same parameter that was found to have the most impact when considering a low effect of parasitism on Ae. triseriatus and no parasite effect on Ae. albopictus. In general, the trends of PRCC values for the parameters are similar to those with a low effect of parasitism on Ae. triseriatus. See Additional file 1: Figure S5.

\section{Dilution effects}

When we consider including dilution effects into parasitism, we find that the equilibrium values appear to be identical to the case without dilution. To ensure comparison of equivalent cases, we find the equilibrium proportion $\hat{P}_{a}$ of Ae. albopictus larvae under dilution. We use this in our dilution formula $\gamma_{i}=\left(\gamma_{\max }-1\right) \hat{P}_{a}+1$ to find comparable parasitism parameters for the absence of dilution. Thus, at our starting condition the parasite parameters in the two cases differ because of the different population proportions, but approach the same values at equilibrium (Fig. 5). In Fig. 5, we plotted four different situations in which we have either a high or low parasite effect for the initial maximum parasite value (Table 3). While the equilibria are identical, there is a difference in the dynamics before reaching equilibrium. This is particularly apparent in the case where Ae. albopictus starts with a high parasite effect. In this case, Ae. triseriatus initially has a greater population before Ae. albopictus becomes the dominant species.

\section{Analytical formulation of the proportion of Ae. albopictus}

We analyzed our model to determine the long-term behavior for the proportion of Ae. albopictus. From the system of equations, there are four possible equilibria: extinction of mosquitoes, competitive exclusion with each species type present, and coexistence. The equilibrium equations can be found in the Additional file 3 . Before we discuss our formula for the population proportion, we introduce the ratio which represents the reproduction number of each species, $R_{t}$ and $R_{a}$, for $A e$.
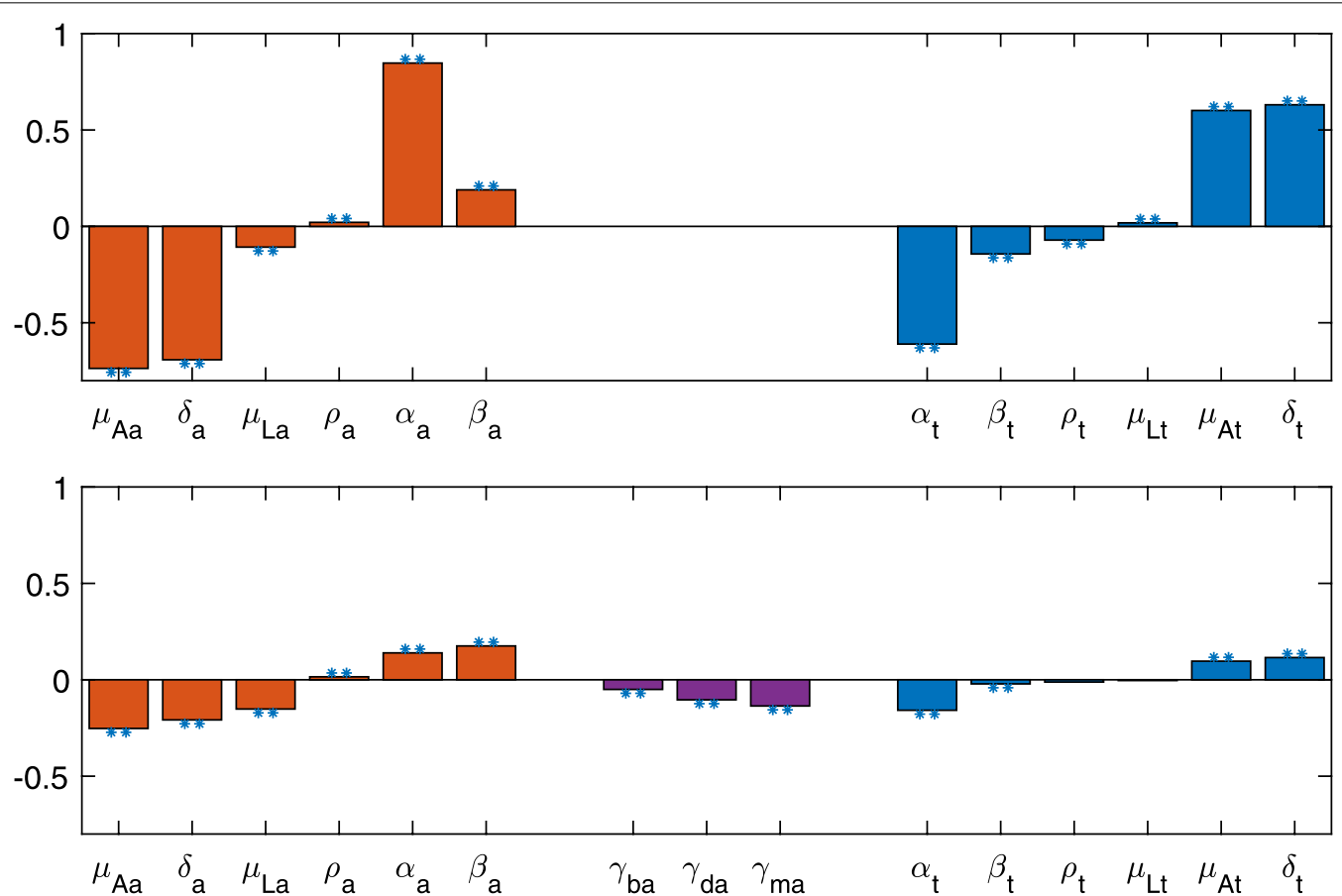

Fig. 4 Partial rank correlation coefficients of the parameter values with the final proportion of Ae. albopictus at 2000 days. The stars indicate significance with a $p$-value $<0.00001$. The top plot shows the PRCC with the LHS with all parasitism parameters set to $1\left(\gamma_{d_{a}}=\gamma_{m_{a}}=\gamma_{b_{a}}=1\right)$, indicating no parasitism. The bottom plot is the PRCC with variation of parasitism included in the LHS for Ae. albopictus. Parasitism on Ae. triseriatus is low. Red bars (left side) are parameters associated with Ae. albopictus, and blue bars (right side) with Ae. triseriatus. Parasitism parameters on Ae. albopictus, which only occur in the bottom plot, are shown by purple bars (middle). For each grouping, parameters are ordered from the least to greatest effect when including parasitism. The PRCC for parameter values with high Ae. triseriatus parasitism is found in Additional file 1: Figure S5 
triseriatus and Ae. albopictus, respectively. These are given by:

$$
\begin{aligned}
R_{a} & =\frac{\beta_{a} \rho_{a}}{\gamma_{b_{a}}} \frac{1}{\mu_{A_{a}}} \frac{\frac{1}{\gamma_{d_{a}} \delta_{a}}}{\frac{1}{\gamma_{d_{a}} \delta_{a}}+\gamma_{m_{a}} \mu_{L_{a}}}, \\
R_{t} & =\beta_{t} \rho_{t} \frac{1}{\mu_{A_{t}}} \frac{\frac{1}{\gamma_{d_{t}} \delta_{t}}}{\frac{1}{\gamma_{d_{t}} \delta_{t}}+\gamma_{m_{t}} \mu_{L_{t}}} .
\end{aligned}
$$

In each reproduction number, the first term is the birth rate, $\frac{\beta_{a} \rho_{a}}{\gamma_{b_{a}}}$, for Ae. albopictus and $\beta_{t} \rho_{t}$ for Ae. triseriatus. This is multiplied by the inverse of the adult death rate, i.e. the expected life span of an adult mosquito. Together, these terms are the total expected number of eggs a female will lay in her lifetime. The final term has the development rate (i.e. $\frac{1}{\gamma_{d_{t}} \delta_{t}}$ and $\frac{1}{\gamma_{d_{a}} \delta_{a}}$, respectively), divided by the sum of the development rate and death rate of larvae. This proportion is the probability of an egg surviving to adulthood. Overall, the reproductive number gives the total surviving children that a single female will lay. If $R_{t}>1$, the population of Ae. triseriatus can establish. Similarly, if $R_{a}>1, A e$. albopictus can establish.

We determine the equilibrium for coexistence in terms of $R_{t}$ and $R_{a}$ (see Additional file 3). From these, we calculate the equilibrium proportion of adult Ae. albopictus by:

$$
\frac{A_{a}}{A_{a}+A_{t}}=\frac{w_{d}\left(1-\alpha_{t}+\frac{\alpha_{t}}{R_{t}}-\frac{1}{R_{a}}\right)}{w_{d}\left(1-\alpha_{t}+\frac{\alpha_{t}}{R_{t}}-\frac{1}{R_{a}}\right)+q_{d}\left(1-\alpha_{a}+\frac{\alpha_{a}}{R_{a}}-\frac{1}{R_{t}}\right)}
$$

where

$$
\begin{aligned}
w_{d} & =\frac{1}{\gamma_{d_{a}} \delta_{a} \mu_{A_{a}}}, \\
q_{d} & =\frac{1}{\gamma_{d_{t}} \delta_{t} \mu_{A_{t}}} .
\end{aligned}
$$

Notice that the parameters $w_{d}$ and $q_{d}$ are the inverse of the quantity of development time multiplied by the adult death rate for Ae. albopictus and Ae. triseriatus, respectively. This represents the expected life span. From our analytical description of the proportion of Ae. albopictus, we can determine the importance of different parameters.

\section{Discussion}

While some studies suggest that Ae. albopictus is the stronger competitor, consistently eggs of both species are found together in the wild even in the presence of high levels of parasitism $[3,7,23,55]$. Indeed, our results show that high levels of infection of Ae. albopictus with As. taiwanensis have a significant effect on the population levels and level the playing field between the two mosquito species.
We find a wide range of situations in which Ae. triseriatus is the dominant species, but primarily in the presence of unrealistically high effects of parasitism in Ae. albopictus (Fig. 3). Thus, it is unlikely that Ae. triseriatus would dominate Ae. albopictus without significant drastic effects of parasitism on the mosquito species. Furthermore, the combined effects of the parasite would likely not result in complete elimination of Ae. albopictus in the wild.

There are additional factors other than the effects of gregarine parasites, that contribute to coexistence. One of these is that the competitive effects are lessened when resources are sufficient [17]. Although Ae. triseriatus develops more slowly and has a smaller survival rate, it can survive at a lower temperature than Ae. albopictus [18]. We also make the assumption that the reproductive cycles and seasons of the two Aedes mosquitoes overlap completely. However, the populations of the two mosquitoes peak at different times: Ae. triseriatus peaks in late June and early July, while Ae. albopictus peaks in late July through late August [3], which could give Ae. triseriatus a slight edge that is not incorporated into this model. Although this is most likely due to other factors, we also saw these dynamics when we considered the dilution of parameters and that Ae. albopictus has a high parasite effect. These temporal changes where Ae. triseriatus initially has a greater population, but then returns to the same coexistence equilibrium, show that the initial seasonal population of $A e$. triseriatus might return to the same equilibrium with the return of Ae. albopictus. It would be possible to study these alterations by changing the initial conditions of the simulations, for example, changing the initial conditions so that Ae. triseriatus starts with both adults and larvae and Ae. albopictus starts only with larvae. Additionally, we could simulate a later introduction of Ae. albopictus to compensate for this difference.

Ae. triseriatus is native to the areas in which it competes with Ae. albopictus and is subsequently unable to escape its parasite in the same way as Ae. albopictus. Consequently, a fixed level parasitism is assumed to occur when the population is at equilibrium. We only consider two levels of infection of Ae. triseriatus by its parasite $A s$. barretti, which affects the development time and mortality of Ae. triseriatus. Thus, we do not consider all possible combinations of parasitism, but it does indicate that the increase of parasitism on Ae. triseriatus increased the proportion of Ae. albopictus, but did not drastically affect the overall dynamics. Additional evidence suggests that Ae. triseriatus that are infected with As. barretti were killed less often by a predator, Toxohrynchites rutilus, compared to uninfected Ae. triseriatus [56]. This shows that while the effect of the parasite does have an effect on the competition between the two species, there are other species 


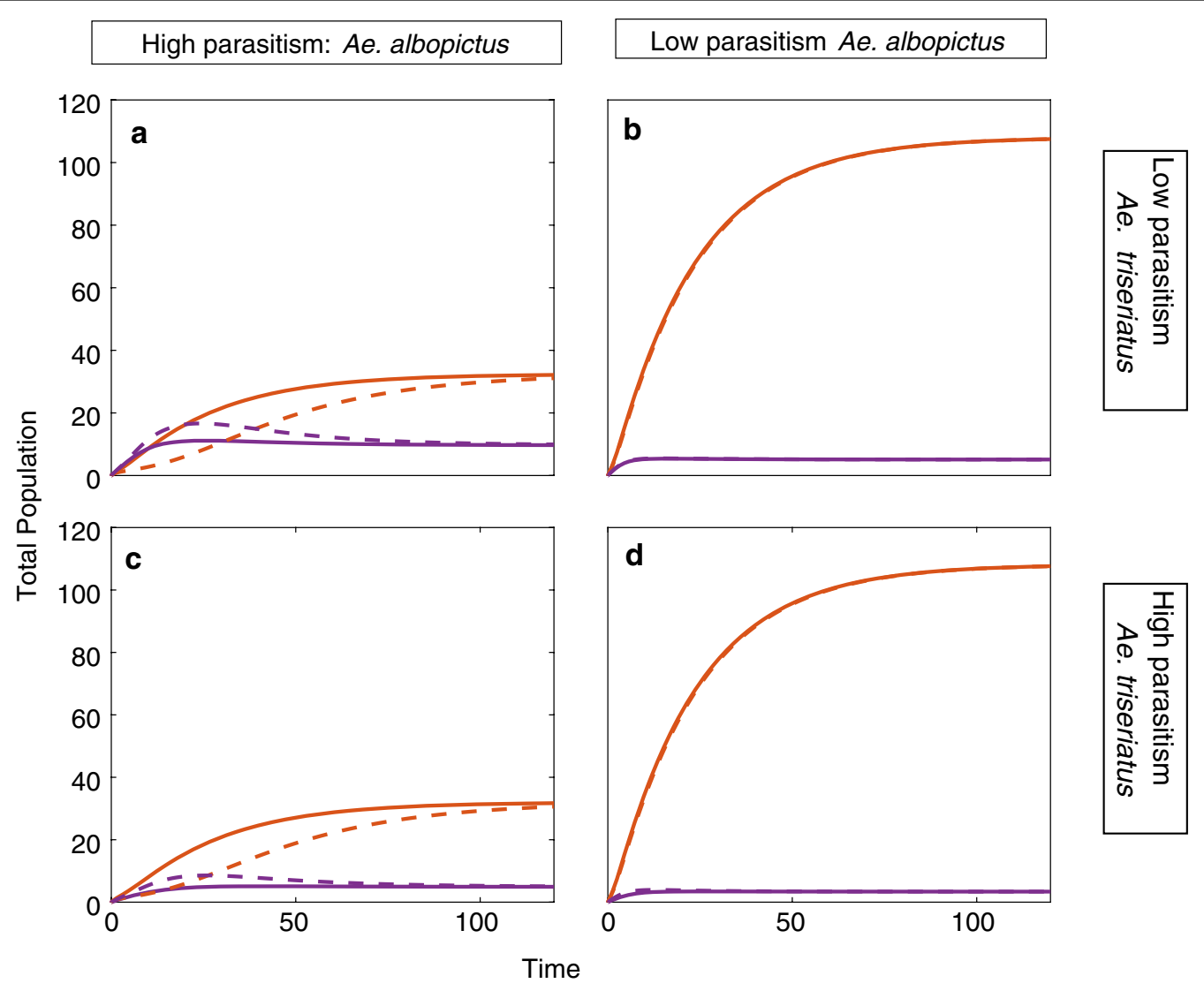

\section{-Dilution effect - Ae. albopictus - - No effect - Ae. albopictus \\ Dilution effect - Ae. triseriatus - - No effect - Ae. triseriatus}

Fig. 5 Dilution effects of parasitism. The proportion of Ae. albopictus when varying the parasitism parameters. All subplots are in the tire scenarios $\left(\alpha_{a}=0.83\right.$ and $\left.\alpha_{t}=0.25\right)$, where the effect of competition on Ae. triseriatus is greater than on Ae. albopictus. The solid lines indicate dilution of parasitism, which means that parasite parameters of a species decrease as their proportion decreases. The dashed lines are in the absence of dilution and assume a constant parasite parameter. See the text for how the values are chosen. The left $(\mathbf{a}, \mathbf{c})$ shows the maximum parasite parameters are larger for Ae. albopictus, and the right $(\mathbf{b}, \mathbf{d})$ has smaller maximum parasite parameters. The top $(\mathbf{a}, \mathbf{b})$ shows smaller maximum parasite parameters for Ae. triseriatus and the bottom $(\mathbf{c}, \mathbf{d})$ larger values. All parameters are listed in Table 3

that might exacerbate or mitigate the effects of As. taiwanensis on Ae. albopictus.

Evidence suggesting that endemic infection of $A e$. albopictus by As. taiwanensis decreases fitness has implications for the spread of mosquito-borne diseases. In southwest Virginia, both Aedes species can act as a vector for La Crosse encephalitis virus (LACV). While Ae. triseriatus is the primary vector for the virus, evidence suggests that as Ae. albopictus becomes more established, it can transmit LACV just as effectively as Ae. triseriatus [11, 12]. Importantly, as Ae. albopictus often occurs in urban or para-urban settings, it can act as a bridge vector for LACV to the human population. While La Crosse encephalitis is not widespread across the US, it is one of the most common mosquito-borne pediatric diseases in the country [57]. LACV infections result in inflammation of the brain, which can lead to seizures and paralysis [2]. In the future, this understanding of mosquito population dynamics can be implemented in the study of mosquitoborne diseases such as La Crosse encephalitis.

\section{Conclusion}

We aimed to determine the extent to which parasitism of Ae. albopictus by As. taiwanensis impacts its competition with Ae. triseriatus. Both Aedes mosquitoes are potentially competent vectors for a number of human arboviruses, and insight into the dynamics of these two species could help inform future disease mitigation efforts. Without parasitism due to As. taiwanensis, Ae. albopictus has a large and distinct competitive advantage over Ae. triseriatus in some environments. As Ae. albopictus is 
an often invasive generalist species, its domination over native Ae. triseriatus in the absence of mitigating effects, such as parasitism, is likely. Our results suggest that the competitive advantage of Ae. albopictus, in the absence of parasitism, is so great that if the species has even the slightest edge over Ae. triseriatus, it will dominate given enough time. From our parameter sweep, $86 \%$ of the scenarios resulted in Ae. albopictus being the dominant species, with few scenarios leading to Ae. triseriatus as the dominant species, under low parasitism on Ae. triseriatus. When we increase the effect of parasitism of $A e$. triseriatus to a high level, Ae. albopictus is dominant in $94 \%$ of the scenarios. Even in treeholes where the competition effect from Ae. triseriatus is greater, Ae. albopictus remains the dominant species. This is consistent with previous results $[7,16,23]$. When considering the impact of parasitism of Ae. albopictus by As. taiwanensis, we observe a decreased overall fitness of the species and negative effects on its competitive ability. Previous empirical results corroborate our findings [7]. To be clear, the reduction of individual traits is assumed, but our results indicate that the overall population levels are significantly affected by changes in these individual traits. This is seen when changing the effect of parasitism (Fig. 3). The three parasitism parameters all have significant effects on the balance between the two species, with the parasite effect on development time having the strongest effect on the proportion of Ae. albopictus. The greatest effect is observed when there is a combined effect on female fecundity, larval development time, and larval mortality. As we increase the effects of the parasite parameters we see that the proportion of Ae. albopictus decreases and leads to elimination of Ae. albopictus when parasitism is extremely high.

\section{Supplementary Information}

The online version contains supplementary material available at https://doi. org/10.1186/s13071-021-04581-0.

Additional file 1. Equilibrium and supplementary figure.

Additional file 2. Code.

Additional file 3. Generated data.

\section{Abbreviations}

Ae.: Aedes; LACV: La Crosse encephalitis virus; PRCC: partial regression correlation coefficients; As.: Ascogregarina; GRR: Gross reproductive rate; GC: Gonotrophic cycle.

\section{Acknowledgements}

Not applicable.

\section{Authors' contributions}

ES developed the model, determined the parameter values, ran initial simulations, developed initial code, and drafted the manuscript. MW developed the model and code, analyzed the model, performed simulations, and revised the manuscript. LC oversaw model development and analysis and edited the manuscript. All authors read and approved the final manuscript.

\section{Funding}

LMC and MW were supported by National Science Foundation (NSF) Standard Grant 1853495. MW was partially supported by a grant from Virginia Tech.

\section{Availability of data and materials}

The datasets generated during the current study are available in its additional files

\section{Ethics approval and consent to participate}

Not applicable.

\section{Consent for publication}

Not applicable.

\section{Competing interests}

The authors declare that they have no competing interests.

\section{Author details}

1 Systems Biology, Virginia Tech, Hahn Hall South Suite 2108, 24061 Blacksburg, VA, USA. ${ }^{2}$ Department of Mathematics, Virginia Tech, 460 McBryde Hall, 225

Stanger Street, 24061 Blacksburg, VA, USA.

Received: 30 July 2020 Accepted: 5 January 2021

Published online: 25 January 2021

References

1. Haddow $A D$, et al. The incidence risk, clustering, and clinical presentation of La Crosse virus infections in the eastern United States, 2003-2007. PLoS One. 2009;4(7):e6145.

2. McJunkin JE, de los Reyes EC, Irazuzta JE, Caceres MJ, Khan RR, Minnich LL, et al. La Crosse encephalitis in children. N Engl J Med. 2001:344(11):801-7.

3. Barker CM, Paulson SL, Cantrell S, Davis BS. Habitat preferences and phenology of Ochlerotatus triseriatus and Aedes albopictus (Diptera: Culicidae) in southwestern Virginia. J Med Entomol. 2003;40(4):403-10.

4. Leisnham PT, Juliano SA. Impacts of climate, land use, and biological invasion on the ecology of immature Aedes mosquitoes: implications for La Crosse emergence. Ecohealth. 2012;9(2):217-28.

5. Westby KM, Fritzen C, Paulsen D, Poindexter S, Moncayo AC. LLa Crosse encephalitis virus infection in field-collected Aedes albopictus, Aedes japonicus, and Aedes triseriatus in Tennessee. J Am Mosq Control Assoc. 2015;31(3):233-41.

6. Benedict MQ, Levine RS, Hawley WA, Lounibos LP. Spread of the tiger: global risk of invasion by the mosquito Aedes albopictus. Vector Borne Zoonotic Dis. 2007;7(1):76-85.

7. Aliabadi BW, Juliano SA. Escape from gregarine parasites affects the competitive interactions of an invasive mosquito. Biol Invasions. 2002;4(3):283-97.

8. Moore CG, Mitchell CJ. Aedes albopictus in the United States: ten-year presence and public health implications. Emerg Infect Dis. 1997;3(3):329.

9. Yee DA, Juliano S, Vamosi SM. Seasonal photoperiods alter developmental time and mass of an invasive mosquito, Aedes albopictus (Diptera: Culi(idae), across its north-south range in the United States. J Med Entomol. 2012;49(4):825-32.

10. Rai KS. Aedes albopictus in the Americas. Annu Rev Entomol. 1991:36(1):459-84.

11. Gerhardt RR, Gottfried KL, Apperson CS, Davis BS, Erwin PC, Smith AB, et al. First isolation of La Crosse virus from naturally infected Aedes albopictus. Emerg Infect Dis. 2001;7(5):807.

12. Westby K, Juliano S. Simulated seasonal photoperiods and fluctuating temperatures have limited effects on blood feeding and life history in Aedes triseriatus (Diptera: Culicidae). J Med Entomol. 2015;52(5):896-906.

13. Ho BC, Ewert A, Chew LM. Interspecific competition among Aedes aegypti, Ae. albopictus, and Ae. triseriatus (Diptera: Culicidae): larval development in mixed cultures. J Med Entomol. 1989;26(6):615-23. 
14. Bevins SN. Invasive mosquitoes, larval competition, and indirect effects on the vector competence of native mosquito species (Diptera: Culicidae). Biol Invasions. 2008;10(7):1109-17.

15. Edgarly JS, Willey MS, LivdahI TP. The community ecology of Aedes egg hatching: implications for a mosquito invasion. Ecol Entomol. 1993;18(2):123-8.

16. Livdahl TP, Willey MS. Prospects for an invasion: competition between Aedes albopictus and native Aedes triseriatus. Science. 1991:253(5016):189-91.

17. Novak MG, Higley LG, Christianssen CA, Rowley WA. Evaluating larval competition between Aedes albopictus and Aedes triseriatus (Diptera: Culicidae) through replacement series experiments. Environ Entomol. 1993;22(2):311-8.

18. Teng HJ, Apperson CS. Development and survival of immature Aedes albopictus and Aedes triseriatus (Diptera: Culicidae) in the laboratory: effects of density, food, and competition on response to temperature. J Med Entomol. 2000;37(1):40-52.

19. Yee DA, Kaufman MG, Juliano SA. The significance of ratios of detritus types and micro-organism productivity to competitive interactions between aquatic insect detritivores. J Anim Ecol. 2007;76(6):1105-15.

20. Juliano SA. Coexistence, exclusion, or neutrality? A meta-analysis of competition between Aedes albopictus and resident mosquitoes. Isr J Ecol Evol. 2010;56(3-4):325-51.

21. Walker ED, Poirier SJ, Veldman WT. Effects of Ascogregarina barretti (Eugregarinida: Lecudinidae) infection on emergence success, development time, and size of Aedes triseriatus (Diptera: Culicidae) in microcosms and tires. J Med Entomol. 1987;24(3):303-9.

22. Munstermann LE, Wesson DM. First record of Ascogregarina taiwanensis (Apicomplexa: Lecudinidae) in North American Aedes albopictus. J Am Mosq Control Assoc. 1990;6(2):235-43.

23. Comiskey NM, Lowrie RC Jr, Wesson DM. Role of habitat components on the dynamics of Aedes albopictus (Diptera: Culicidae) from New Orleans. J Med Entomol. 1999;36(3):313-20.

24. Beier JC, Harris C. Ascogregarina barretti (Sporozoa: diplocystidae) infections in natural populations of Aedes triseriatus (Diptera: Culicidae). J Parasitol. 1983;69(2):430.

25. Siegel JP, Novak RJ, Maddox JV. Effects of Ascogregarina barretti (Eugregarinida: Lecudinidae) infection on Aedes triseriatus (Diptera: Culicidae) in Illinois. J Med Entomol. 1992;29(6):968-73.

26. de Assis RA, Casacci LP, Bonelli S, Barbero F, de Assis LME, Venturino E. Effects of host interspecific interaction in the maculinea-myrmica parasite-host system. Bull Math Biol. 2018;80(9):2378-407.

27. Gilpin W, Feldman MW, Aoki K. An ecocultural model predicts Neanderthal extinction through competition with modern humans. PNAS. 2016;113(8):2134-9.

28. Liu R, Liu G. Asymptotic behavior of a stochastic Two-Species competition model under the effect of disease. Complexity. 2018. doi:10.1155/2018/3127404

29. Sebastián-González E, Moleón M, Gibert JP, Botella F, Mateo-Tomás P, Olea PP, et al. Nested species-rich networks of scavenging vertebrates support high levels of interspecific competition. Ecology. 2016;97(1):95-105.

30. Tan X, Gu H, Ruan Y, Zhong J, Parajuli K, Hu J. Effects of nitrogen on interspecific competition between two cell-size cyanobacteria: Microcystis aeruginosa and Synechococcus sp. Harmful Algae. 2019:89:101661.

31. Zhou P, Zhao XQ. Global dynamics of a two species competition model in open stream environments. J Dyn Differ Equ. 2018;30(2):613-36.

32. Bewick S, Agusto F, Calabrese JM, Muturi EJ, Fagan WF. Epidemiology of La Crosse virus emergence, Appalachia Region, United States. Emerg Infect Dis. 2016;22(11):1921.

33. Marini G, Guzzetta G, Baldacchino F, Arnoldi D, Montarsi F, Capelli G, et al. The effect of interspecific competition on the temporal dynamics of Aedes albopictus and Culex pipiens. Parasit Vectors. 2017;10(1):102.

34. Paton RS, Bonsall MB. The ecological and epidemiological consequences of reproductive interference between the vectors Aedes aegypti and Aedes albopictus. J R Soc Interface. 2019;16(156):20190270.

35. Kuno E. Competitive exclusion through reproductive interference. Popul Ecol. 1992:34(2):275-84
36. Kishi S, Nakazawa T. Analysis of species coexistence co-mediated by resource competition and reproductive interference. Popul Ecol. 2013:55(2):305-13.

37. Chen W. The life cycle of Ascogregarina taiwanensis (Apicomplexa: Lecudinidae). Parasitol Today. 1999;15(4):153-6.

38. Prophiro JS, Pereira TN, Oliveira JGd, Dandolini GW, Silva MANd, Silva OSd. Ascogregarina taiwanensis infection in Aedes aegypti and Aedes albopictus in Santa Catarina, South Brazil. Rev Soc Bras Med Trop. 2017;50(2):235-8.

39. Lotka AJ. Analytical note on certain rhythmic relations in organic systems. PNAS. 1920;6(7):410-5.

40. Lotka AJ. Contribution to the theory of periodic reactions. J Phys. 2002;14(3):271-4.

41. Smith TM, Smith RL. Elements of ecology. Ninth edn. Boston:Pearson; 2015.

42. Volterra V. Variations and fluctuations of the number of individuals in animal species living together. ICES J Mar Sci. 1928;3(1):3-51.

43. Erickson RA, Presley SM, Allen LJ, Long KR, Cox SB. A stage-structured, Aedes albopictus population model. Ecol Model. 2010;221(9):1273-82.

44. Delatte H, Gimonneau G, Triboire A, Fontenille D. Influence of temperature on immature development, survival, longevity, fecundity, and gonotrophic cycles of Aedes albopictus, vector of Chikungunya and dengue in the Indian Ocean. J Med Entomol. 2009;46(1):33-41.

45. Jalil M. Observations on the fecundity of Aedes triseriatus (Diptera: Culicidae). Entomol Exp Appl. 1974;17(2):223-33.

46. Mather TN, DeFoliart GR. Effect of host blood source on the gonotrophic cycle of Aedes triseriatus. Am J Trop Med Hyg. 1983;32(1):189-93.

47. Briegel H, Timmermann SE. Aedes albopictus (Diptera: Culicidae): physiological aspects of development and reproduction. J Med Entomol. 2001;38(4):566-71.

48. Maimusa HA, Ahmad AH, Kassim NFA, Rahim J. Age-stage, two-sex life table characteristics of Aedes albopictus and Aedes Aegypti in Penang Island. Malaysia. J Am Mosq Control Assoc. 2016;32(1):1-11.

49. Haramis LD, Foster WA. Survival and population density of Aedes triseriatus (Diptera: Culicidae) in a woodlot in central Ohio, USA. J Med Entomol. 1983;20(4):391-8

50. Patrican L, DeFoliart G. Lack of adverse effect of transovarially acquired La Crosse virus infection on the reproductive capacity of Aedes triseriatus (Diptera: Culicidae). J Med Entomol. 1985;22(6):604-11.

51. Soghigian J, Livdahl T. Differential response to mosquito host sex and parasite dosage suggest mixed dispersal strategies in the parasite Ascogregarina taiwanensis. PloS One. 2017;12(9):e0184573.

52. Helton JC, Davis FJ. Latin hypercube sampling and the propagation of uncertainty in analyses of complex systems. Reliab Eng. 2003;81(1):23-69.

53. Marino S, Hogue IB, Ray CJ, Kirschner DE. A methodology for performing global uncertainty and sensitivity analysis in systems biology. J Theor Biol. 2008;254(1):178-96

54. Westby KM, Sweetman BM, Van Horn TR, Biro EG, Medley KA. Invasive species reduces parasite prevalence and neutralizes negative environmental effects on parasitism in a native mosquito. J Anim Ecol. 2019;88(8):1215-25

55. Dixson A, Jackson R, Rowe R, Nease R, Fryxell RT. Aedes albopictus oviposits with other Aedes species in artificial oviposition cups: a case study in Knox County, Tennessee, USA. J Vector Ecol. 2020:45(1):2-15.

56. Soghigian J, Valsdottir LR, Livdahl TP. A parasite's modification of host behavior reduces predation on its host. Ecol Evol. 2017;7(5):1453-61.

57. Rust RS, Thompson WH, Matthews CG, Beaty BJ, Chun RW. Topical review: La Crosse and other forms of California encephalitis. J Child Neurol. 1999;14(1):1-14.

\section{Publisher's Note}

Springer Nature remains neutral with regard to jurisdictional claims in published maps and institutional affiliations. 